\title{
Introduction for Volume 9, Issue 2
}

This issue contains 6 papers. There are five contributions in English and one contribution in Chinese with English abstracts. The papers can be divided into five topics: disaster response, earthquake risk assessment, ecological environment risk management, urban vulnerability assessment and agricultural weather insurance.

Concerning to disaster response, the paper "Competency of the Infantry Troops of the Nepalese Army in Disaster Response” by Shrijan Bahadur Malla, et al., analyses the Nepalese Army's disaster response's soft skill knowledge, technical skill knowledge and preparedness activities along with the perception of senior army officers.

There are two papers earthquake risk assessment. The paper "Seismic Hazard Model Harmonization in Tienshan Area" by Changlong Li, Mengtan Gao, provides a comparison between Central Asia, the Middle East hazard models and the Chinese hazard result in Tienshan Area, based on reshaped seismogenic sources, recalculated related seismicity parameters, and calibrated ground motion models. This paper concluded that in the most areas of Tienshan, the Peak Ground Acceleration (PGA) with $10 \%$ probability of exceedance in 50 years is around $0.2 \mathrm{~g} \sim 0.3 \mathrm{~g}$, and northern part of Pakistan has the highest seismic hazard in Tienshan Area. The second paper "Applicability of Regional Evaluation for Rapid Assessment Models of Earthquake Disaster Life Loss -A case study of Gansu Province" by Wen Li, et al., uses the typical earthquake disaster life loss assessment models to verify and calculate the historical earthquake cases in the Gansu Province since 1966. The assessment accuracy and applicability of various models in the Gansu Province are studied by means of actual earthquake cases. Results show that: (1) For $\mathrm{Ms}<5.5$ and $5.5 \leq \mathrm{Ms} \leq 6.0$, the life loss assessment model based on population density proposed by Badal, the life loss assessment model based on epicentral intensity proposed by Liu Jinlong, and the national earthquake disaster emergency assessment model are relatively accurate and basically included in the reasonable range; for $\mathrm{Ms}>6.0$, characteristics of the assessed regions, such as physical geography and social economy, should be analyzed to correct the model calculation results and to receive comprehensive assessment results, so as to support emergency decisions. (2) Earthquake disaster life loss assessment models are regionalized and the assessment results are obviously regional. Assessment results of the earthquakes occurred in Hedong Region are not accurate for Hexi Region of Gansu Province. In the studies on earthquake disaster life loss assessment models, development of the typical assessment models targeted on life loss characteristics of regional earthquakes has practical significance to actual earthquake emergency response and it is convenient to apply scientific research results in practical work.

There is one paper in ecological environment risk management. The paper "Evaluation of Guizhou Ecological Civilization Construction Level Based on Intuitionistic Fuzzy Analytic Hierarchy Process” by Fangping Wang, et al., calculates the nine province each secondary index to the weight of a layer and target layer by using IFAHP, the establishment of intuitionistic fuzzy judgment matrix and consistency test. Comprehensive evaluation value is calculated and sorted. The empirical research shows that the ecological civilization construction level of Guizhou province is relatively high, but the level distribution of all prefectures and cities is very uneven, and the difference between the highest score southeast Guizhou and the lowest score Liupanshui is 4.87. Therefore, it is necessary to accelerate the construction of ecological civilization in various prefectures and cities, so as to improve the construction level of ecological civilization in the whole province and promote regional economic development.

There is also one paper in urban vulnerability assessment. The paper "Spatial and Temporal Patterns of Urban Vulnerability in Guangzhou” by Bo Tang, et al., develops an urban vulnerability index system from four aspects of population vulnerability, economic vulnerability, social vulnerability, and ecological vulnerability. The Spatial and temporal patterns of urban Vulnerability in Guangzhou were evaluated by a comprehensive index model and GIS analysis from three time sections $(2005 、 2010 、 2014)$. The results indicated that: (1) economic density, population density, and per capita GDP are the main factors affecting the vulnerability of Guangzhou. (2) The population 
vulnerability is considerably higher for the central group cities than for the peripheral group cities. The economic vulnerability reflects a crisscross pattern, consisting of a central group and a peripheral group. The social and ecological vulnerabilities are higher for the peripheral group than for the central group. (3) The comprehensive vulnerability has a layered structure, with a high vulnerability in the inner ring layer, low vulnerability in the middle layer, and medium vulnerability in the outer layer.

There is one paper in agricultural weather insurance. The paper "Summary of Researches on Basis Risk in Weather Index Insurance" by Wang Yueqin, et al., summarizes researches on the theories and practices of weather index insurance at home and abroad, and systematically comments on the basis risk's definition, formation mechanism, quantitative methods and management measures. In order to provide useful ideas and references for the development of China's weather index insurance and the future research on basis risk.

We sincerely thank the referees for their strong support and kind help. Thanks to all the authors for their submissions. Particularly, thanks to Prof. Mu Zhang, Publication Chair of the Society for Risk Analysis - China, and thanks to Prof. Junxiang Zhang, Manager of Journal of Risk Analysis and Crisis Response, they devoted their time to overseeing the reviews.

\section{Editors-in-Chief}

\author{
Prof. Chongfu Huang \\ Beijing Normal University \\ No.19 Xinjiekouwai Street \\ Beijing 100875, China \\ Email: hchongfu@126.com
}

\section{Prof. Gordon Huang}

Faculty of Engineering and Applied Science,

University of Regina

Regina, Sask S4S 0A2, Canada

Email: gordon.huang@uregina.ca 